\title{
Young Star Clusters in the Small Magellanic Cloud: Impact of Local and Global Conditions on Star Formation
}

\author{
Elena Sabbi ${ }^{1}$, Linda J. Smith ${ }^{1,2}$, Lynn R. Carlson ${ }^{3}$, Antonella Nota ${ }^{1,4}$, \\ Monca Tosi ${ }^{5}$, Michele Cignoni ${ }^{5}$, Jay S. Gallagher III $^{6}$, \\ Marco Sirianni $^{1,4}$, and Margaret Meixner ${ }^{1}$ \\ ${ }^{1}$ Space Telescope Science Institute \\ 3700 San Martin Drive, Baltimore, MD, 21218, USA \\ email: sabbi@stsci.edu \\ ${ }^{2}$ University College London \\ London, UK \\ ${ }^{3}$ Johns Hopkins University \\ Baltimore, MD, USA \\ ${ }^{4}$ European Space Agency, Research and Scientific Support Department \\ Baltimore, MD, USA \\ ${ }^{5}$ INAF-Osservatorio Astronomico di Bologna \\ Bologna, Italy \\ ${ }^{6}$ University of Wisconsin \\ Madison, WI, USA
}

\begin{abstract}
We compared deep images acquired with the Advanced Camera for Surveys on board of the Hubble Space Telescope with mid-IR Spitzer Space Telescope images and University College London Echelle Spectrograph spectra of NGC 346 and NGC 602, two of the youngest star clusters in the Small Magellanic Cloud. Our multi-wavelength approach allowed us to infer very different origins for the clusters: while NGC 346 is likely the result of the hierarchical collapse of a giant molecular cloud, NGC 602 is probably the result of the collision and consequent interaction of two $\mathrm{H}$ I shells of gas.
\end{abstract}

Keywords. Magellanic Clouds, galaxies: star clusters, stars: formation, stars: mass function, stars: pre-main-sequence

\section{Introduction}

As the closest star forming dwarf galaxy $(\sim 60 \mathrm{kpc})$, the Small Magellanic Cloud (SMC) represents an ideal laboratory for detailed studies of resolved stellar populations in this extremely common class of objects. In addition its present day metallicity $(Z=0.004)$ and low dust content (1/30 the Milky Way) make the SMC the best local analog to the vast majority of late-type dwarfs.

Deep images acquired with the Advanced Camera for Surveys (ACS) on board of the Hubble Space Telescope (HST) provide excellent photometry well below the turnoff (TO) of the oldest stellar population in the SMC, allowing us to infer an accurate star formation history (SFH) over the entire Hubble time. Furthermore the high spatial resolution of ACS allowed us to spatially resolve even the densest star cluster in the SMC to probe the cluster formation and evolution in late-type dwarf galaxies.

The availability of multi-wavelength surveys from the radio band to the far-IR, combined with the simple kinematics of the galaxy allow us to identify the possible triggers 
of star formation (SF), while using high resolution spectroscopy we can study the effect of reduced stellar wind power on the early cluster evolution.

With the aim of understanding the impact of global and local conditions on early star cluster evolution we recently acquired deep F555W $(\sim \mathrm{V})$ and F814W $(\sim$ I $)$ ACS Wide Field Channel (WFC) images of two of the youngest SMC star clusters: NGC 346 and NGC 602.

\section{Photometric properties of the star clusters}

$\underline{\text { NGC } 346}$ is the most massive star forming region of the SMC, and is located in the bar of the SMC, a region characterized by high gas and stellar densities. The cluster contains half of the known $\mathrm{O}$ stars in the SMC, and is ionizing the bright nebula N66.

A first inspection of the NGC $346 m_{\mathrm{F} 555 \mathrm{~W}}$ vs. $m_{\mathrm{F} 555 \mathrm{~W}}-m_{\mathrm{F} 814 \mathrm{~W}}$ color magnitude diagram (CMD, Fig. 1-left panel) reveals that different stellar populations are present in this area:

- Young stars. The bright $\left(12.5 P \lesssim m_{\mathrm{F} 555 \mathrm{~W}} \lesssim 22\right)$ and blue $\left(0.3 \lesssim m_{\mathrm{F} 555 \mathrm{~W}}-\right.$ $\left.m_{\mathrm{F} 814 \mathrm{~W}} \lesssim 0.4\right)$ main sequence $(\mathrm{MS})$, well visible to the upper left of the CMD, as well as the sequence of red $\left(1.5 \lesssim m_{\mathrm{F} 555 \mathrm{~W}}-m_{\mathrm{F} 814 \mathrm{~W}} \lesssim 2.2\right)$ and faint $\left(m_{\mathrm{F} 555 \mathrm{~W}} \lesssim 21\right)$ pre-MS stars in the mass range $0.6-3 M_{\odot}$ are representative of a stellar population that formed between 3 and 5 Myr ago (Nota et al. 2006, Sabbi et al. 2007).

- Intermediate-age and old stars. An older stellar population is easily distinguishable in the CMD. Its rich $\mathrm{MS}$ extends from $m_{\mathrm{F} 555 \mathrm{~W}} \simeq 22$ down to $m_{\mathrm{F} 555 \mathrm{~W}} \simeq 26.5$. The evolved phases of this population are well delineated: the narrow subgiant branch (SGB) $\left(m_{\mathrm{F} 555 \mathrm{~W}} \simeq 21.6 ; 0.45 \lesssim m_{\mathrm{F} 555 \mathrm{~W}}-m_{\mathrm{F} 814 \mathrm{~W}} \lesssim 0.95\right)$; the very well defined red giant branch (RGB) (with the brightest stars at $m_{\mathrm{F} 555 \mathrm{~W}} \simeq 17.3$ and $m_{\mathrm{F} 555 \mathrm{~W}}-m_{\mathrm{F} 814 \mathrm{~W}} \simeq 0.65$ ), and the red clump $(\mathrm{RC})\left(m_{\mathrm{F} 555 \mathrm{~W}} \simeq 19.5\right)$. Isochrones fitting indicates that the majority of the older stars in this field formed in a single SF episode approximately 4.5 Gyr ago (Sabbi et al. 2007).

Figure 2 shows the present day mass function (PDMF) of NGC 346 (open circles) between 0.8 and $60 \mathrm{M}_{\odot}$. In this range of masses the slope of the NGC 346 PDMF $\Gamma=-1.43 \pm 0.18$ (Sabbi et al. 2008), in good agreement with the value derived by Salpeter $(1955)$ for the solar neighborhood $\left(\Gamma_{\text {Salpeter }}=-1.35\right)$.

NGC 602 is a small and bright star forming region, located between the SMC wing and the Magellanic bridge, a region characterized by low gas and dust content. Figure 1-right panel shows NGC $602 m_{\mathrm{F} 555 \mathrm{~W}}$ vs. $m_{\mathrm{F} 555 \mathrm{~W}}-m_{\mathrm{F} 814 \mathrm{~W}} \mathrm{CMD}$. The most striking feature of this CMD is the rich population of pre-MS stars in the mass range 0.6-3 $\mathrm{M}_{\odot}$ (Carlson et al. 2007). The TO near $m_{\mathrm{F} 555 \mathrm{~W}} \simeq 22$, the $\mathrm{SGC}$ also at $m_{\mathrm{F} 555 \mathrm{~W}} \simeq 22$, and the $\mathrm{RC}$ around $m_{\mathrm{F} 555 \mathrm{~W}} \simeq 19.5$ belong likely to the SMC field stellar population that is present even in this low stellar density region. Isochron fitting indicates an age of $\sim 4$ Myr for NGC 602 (but see the poster presented in this conference by Cignoni et al. for a detailed SFH history of this region).

We derived the PDMF of NGC 602 in the mass range $0.8-30 \mathrm{M}_{\odot}$ (Fig.2-filled ellipses). From the weighted least mean squares fit of the data we derived a slope $\Gamma=-1.25 \pm 0.22$ (Cignoni et al. 2008b) in excellent agreement with what we found in NGC 346.

Does the universality of the MF imply that the SF process is independent of both the local and global conditions? 


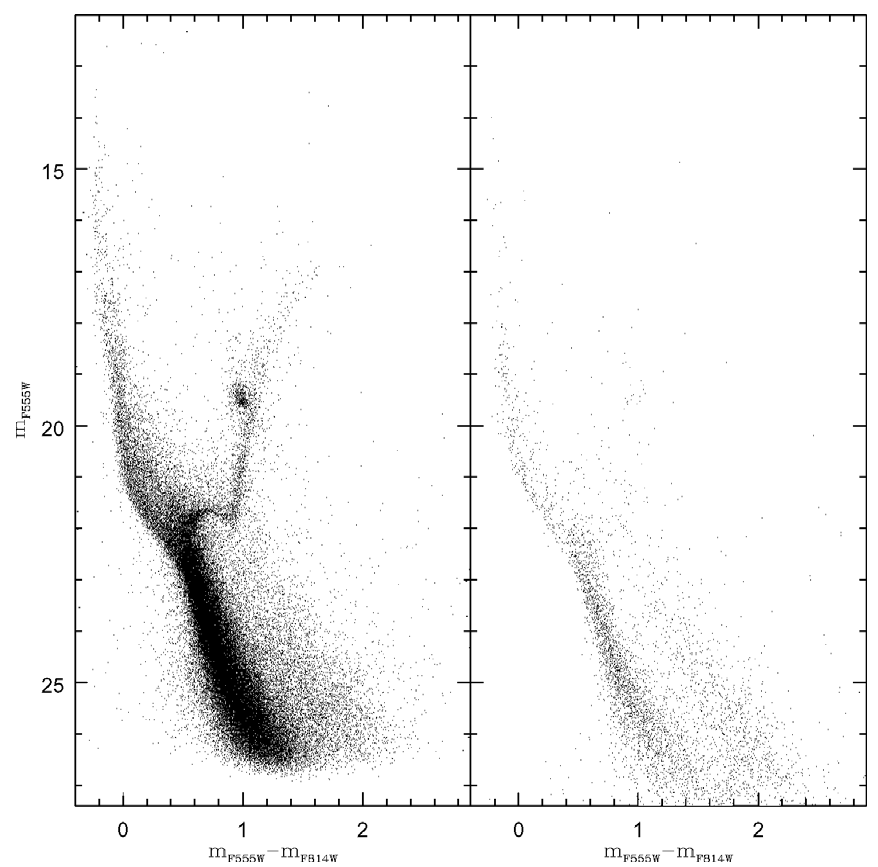

Figure 1. ACS/WFC $m_{\mathrm{F} 555 \mathrm{~W}}$ vs. $m_{\mathrm{F} 555 \mathrm{~W}}-m_{\mathrm{F} 814 \mathrm{~W}} \mathrm{CMDs}$ of NGC 346 (left panel) and NGC 602 (right panel).

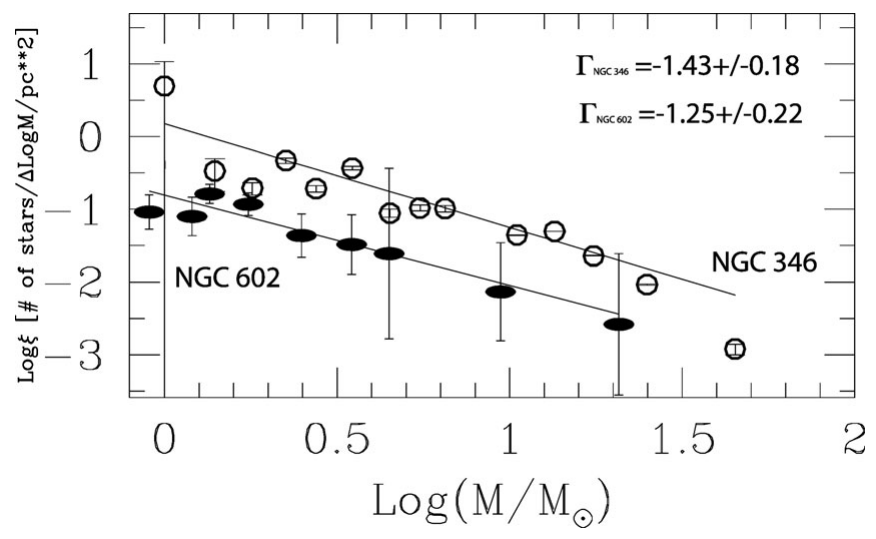

Figure 2. PDMFs of NGC 346 (open circles) between 0.8 and $60 \mathrm{M}_{\odot}$ and of NGC 602 (filled ellipses) between 0.8 and $30 \mathrm{M}_{\odot}$.

\section{NGC 346 structure and kinematics}

The high spatial resolution of ACS/WFC showed that the NGC 346 stars are organized in a number of small compact sub-clusters, which vary in density, dust content, and morphology, but, within the uncertainties of isochrone fitting, are likely coeval. The majority of these associations are still embedded in HII gas, and dust. Arcs and filaments of dust and gas depart from the most central sub-clusters and connect them with the external associations (Sabbi et al. 2007).

The sub-clusters are coincident with the CO clumps described by Rubio et al. (2000). Recent Spitzer Space Telescope (SST) mid-IR observations also revealed that all the sub-clusters contain one or more young stellar objects (YSOs) with ages younger than 
1 Myr (Simon et al. 2007). By assuming a Salpeter IMF, Simon et al. (2007) calculated that more than $3000 \mathrm{M}_{\odot}$ have been formed in the last $\sim 1 \mathrm{Myr}$, concluding that $6 \%$ of the current $\mathrm{SF}$ in the $\mathrm{SMC}$ is taking place in NGC 346.

The fact that the stars are organized in sub-clusters, connected by filaments of dust and gas, and that star formation is still ongoing in the sub-clusters suggest that NGC 346 is the result of the collapse and subsequent fragmentation of an initial giant molecular cloud into multiple "seeds" of SF, and may represent a good observational match to the conditions predicted by the hierarchical fragmentation of a molecular cloud in a turbulent interstellar medium (Klessen \& Burket 2000; Bonnell \& Bate 2002; Bonnell et al. 2003). According to this model, the fragmentation of the cloud is due to supersonic turbulent motions present in the gas. The turbulence induces the formation of shocks in the gas and produces filamentary structures (Bate et al. 2003). The chaotic nature of the turbulence increases the density in the filamentary structures locally. When regions of high density become self-gravitating, they start to collapse to form stars. Simulations show that SF occurs simultaneously at several different locations in the cloud (Bonnell et al. 2003), as appears to be the case for NGC 346 .

The formation of shocks in the gas, due to the initial supersonic turbulence, should rapidly remove kinetic energy from the gas (Ostriker et al. 2001). Therefore to further confirm the origin of NGC 346, we observed many sub-clusters with the University College London Echelle Spectrograph (UCLES) at the Anglo-Australian Telescope. We find an average radial velocity $v_{\text {rad }}=165.2 \mathrm{~km} / \mathrm{s}$, with a dispersion $\sigma=1.9 \mathrm{~km} / \mathrm{s}$, further supporting the hypothesis that NGC 346 is the result of a hierarchical collapse. Our spectroscopic analysis also indicates that the ionized gas is quiescent, with no evidence of large-scale motions, confirming that the mechanical feedback is reduced at low metallicity (see the discussion in Mokiem et al. 2007), and proving that supernovae have not yet exploded (Smith et al. - in preparation).

\section{NGC 602 kinematics}

In the ACS/WFC images NGC 602 appears as a small star cluster surrounded by two dusty arches, characterized by numerous dark "pillars of creations". SST observations revealed numerous class 0.5 and I YSOs on the verge of the pillars, indicating that SF propagated from the center of NGC 602, and a second generation of stars is currently forming in the periphery of the cluster (Carlson et al. 2007).

Even if the morphology of this association is reminiscent of an expanding bubble of gas, UCLES high-resolution spectra show very low velocity dispersion, excluding large-scale motions (Nigra et al. 2008). Another possible explanation for the NGC 602 morphology is that we are observing a cavity eroded by the OB UV stellar radiation.

NGC 602 and its associated HII region, N90, formed in a relatively isolated and diffuse environment. Its isolation from other regions of massive star formation and the relatively simple surrounding Hi shell structure encouraged us to try to constrain the processes that may have led to its formation. Using the shell catalog derived from the $21 \mathrm{~cm}$ neutral hydrogen (HI) spectrum survey data (Staveley-Smith et al. 1997; Staminirovíc et al. 1999) we identified a distinct Hi cloud component that is likely the progenitor cloud of the cluster and the HiI region which probably formed in blister fashion from the cloud's periphery. A comparison between Hi and HII kinematics suggests that star formation in NGC 602 was triggered by compression and turbulence associated with HI shell interaction $\sim 7$ Myr ago (Nigra et al. 2008). 


\section{Conclusions}

We have recently analyzed the two very young SMC star clusters NGC 346 and NGC 602, which are located in two regions characterized by very different gas and stellar densities. We derived the PDMF of the clusters over two orders of magnitude, further confirming its universality.

Both clusters contain noticeable populations of pre-MS stars and YSOs, indicating that in both cases SF is still ongoing and residual gas is still present. The ionized gas is quiescent, and we did not find evidence of stellar wind interaction, confirming the hypothesis that mechanical feedback is reduced at low metallicities.

Our multi-wavelength approach allowed us to infer very different origins for the clusters, suggesting that different local condition might deeply affect the formation and the evolution of star clusters since the earliest phases.

\section{References}

Bonnell, I. A. \& Bate, M. R. 2002, MNRAS, 336, 659

Bonnell, I. A., Bate, M. R., \& Vine, S. G. 2003, MNRAS, 343, 413

Cignoni, M. et al. 2008, Proceedings IAU Symposium No. 255, "Low-Metallicity Star Formation: From the Firts Stars to Dwarf Galaxies", L.K. Hunt, S. Madden, \& R. Schneider, eds.

Cignoni, M. et al. 2008, AJ, (submitted)

Klessen, R. S. \& Burkert, A. 2000, ApJS, 128, 287

Mokiem, M. R., et al. 2007, A\&A, 473, 603

Nigra, L., Gallagher, J. S., III, Smith, L. J., Stanimirović, S., Nota, A., \& Sabbi, E. 2008, PASP, (accepted for publication) astro-ph/0808.1033

Nota, A. et al. 2006, ApJ, 640, L29

Ostriker, E. C., Stone, J. M., \& Gammie, C. F. 2001, ApJ, 546, 980

Rubio et al. 2000, A\&A

Sabbi, E., Sirianni, M., Nota, A., Tosi, M., Gallagher, J., Meixner, M., Oey, M. S., Walterbos, R., Pasquali, A., Smith, L.J., \& Angeretti, L. 2007, AJ, 133, 44

Sabbi, E., Sirianni, M., Nota, A., TOsi, M., Gallagher, J. Smith, L. J., Angeretti, L., Oey, M. S., Walterbos, R., \& Pasquali, A. 2008, AJ, 135, 173

Simon, J. H., et al. 2007, ApJ, 670, 313

Stanimirović, S., Staveley-Smith, L., Dickey, J. M., Sault, R. J., \& Snowden, S. L. 1999, MNRAS, 302,417

Staveley-Smith, L., Sault, R. J., Hatzidimitriou, D., Kesteven, M. J., \& McConnell, D. 1997, MNRAS, 289, 225 